\title{
Inverse Meyer-Neldel behavior for activated processes in model glasses
}

\author{
Pawel Koziatek, ${ }^{1,2}$ Jean-Louis Barrat, ${ }^{2}$ Peter Derlet, ${ }^{3}$ and David Rodney ${ }^{1}$ \\ ${ }^{1}$ Science et Ingénierie des Matériaux et Procédés, Grenoble INP, CNRS/UJF, 38402 Saint Martin d'Hères, France \\ ${ }^{2}$ Laboratoire Interdisciplinaire de Physique, Université Joseph Fourier Grenoble, CNRS, 38402 Saint-Martin d'Hères, France \\ ${ }^{3}$ Condensed Matter Theory Group, Paul Scherrer Institute, 5232 Villigen PSI, Switzerland
}

(Received 29 April 2013; revised manuscript received 27 May 2013; published 13 June 2013)

\begin{abstract}
The activation-relaxation technique is used to explore the distribution of escape times from metastable minima of a model metallic glass, estimated using the harmonic transition state theory. We investigate in particular the distribution of attempt frequencies and find that these prefactors are distributed over a range of almost ten orders of magnitude, in stark contrast with the common assumption that they are given by a typical vibrational frequency. Moreover, the typical attempt frequencies appear to decrease with increasing barrier energy, a behavior inverse to the so-called Meyer-Neldel rule observed in many processes with apparent Arrhenius behavior. When combined with a multiple-hop analysis that takes into account the multiplicity of possible transitions, a direct Meyer-Neldel behavior is recovered, albeit with a reduced characteristic prefactor.
\end{abstract}

DOI: 10.1103/PhysRevB.87.224105

PACS number(s): 61.43.-j, 63.50.Lm

Thermally activated processes are ubiquitous in the structural relaxation and deformation of glasses at low temperatures. The onset of the thermally activated regime has been the subject of numerous studies based on molecular dynamics (MD) simulations. ${ }^{1}$ It was shown that the dynamics becomes dominated by thermally activated transitions between local equilibrium configurations [also called inherent structures (ISs)] when the temperature decreases below the modecoupling temperature $\left(T_{C}\right)^{2}$ In this regime, MD simulations become inefficient because the waiting time between transitions increases exponentially with decreasing temperature and rapidly exceeds the MD time scale. An alternative approach is to use the transition state theory (TST), ${ }^{3}$ which expresses the rate of escape from an initial equilibrium state as

$$
\Gamma=v_{\text {att }} \exp \left(-\frac{E^{\star}-E^{0}}{k T}\right),
$$

where $E_{A}=E^{\star}-E^{0}$ is the activation energy, $E^{0}$ the energy of the initial state, and $E^{\star}$ the energy of the activated state, the minimum energy state along the dividing surface between the basins of attraction of the initial and final states. Using a classical harmonic approximation of the TST (hTST), ${ }^{4}$ the attempt frequency, $v_{\text {att }}$, is expressed as

$$
v_{\mathrm{att}}^{\mathrm{hTST}}=\frac{\prod_{i=1}^{3 N-3} v_{i}^{0}}{\prod_{i=1}^{3 N-4} v_{i}^{\star}},
$$

where $\left\{v_{i}^{0}\right\}$ and $\left\{v_{i}^{\star}\right\}$ are the real nonzero eigenfrequencies of the equilibrium and activated states, respectively, computed by diagonalizing the Hessian matrix of the system in both states. Here, we implicitly consider a system of $N$ atoms with periodic boundary conditions. The three degrees of translational invariance are then omitted in Eq. (2) and there is one less frequency in the activated state because of the imaginary frequency associated with the unstable mode.

Activated states are identified here using the activationrelaxation technique (ART), initially proposed by Mousseau and co-workers, ${ }^{5}$ which allows one to explore the configuration space around equilibrium configurations in search of activated states, starting in random directions. In the case of disordered solids, an exhaustive search is unfeasible because of the exponential number of saddle points. However, it has been shown that ART can identify enough saddles to build statistically relevant samples, from which stationary distributions can be computed. ${ }^{6-8}$

So far, attempt frequencies have received far less attention than activation energies and are usually taken as a constant independent of the process and on the order of $1 \times 10^{12}$ to $1 \times$ $10^{13} \mathrm{~s}^{-1}$, i.e., a typical Debye frequency. In reality, a number of phenomena that are governed by an apparent Arrheniuslike temperature dependence have been shown to exhibit a dependence of the attempt frequency on activation energy, when some external parameter (e.g., composition, pressure, or annealing conditions) is varied. This correlation, observed for a wide range of phenomena, is referred to as the Meyer-Neldel compensation rule. ${ }^{9}$ It is expressed as

$$
v_{\text {att }}=v_{\text {att }}^{0} \exp \left(\frac{E_{A}}{k T_{0}}\right)^{\alpha},
$$

with $k T_{0}$ a characteristic energy and $\alpha$ an exponent usually taken as $1 .{ }^{10}$ Theoretical models interpret this relation based on two different processes depending on the situation: (1) thermal activation into exponentially distributed energy traps for diffusion in disordered systems ${ }^{11}$ and (2) entropy of combining multiple thermal excitations to overcome high-energy barriers. ${ }^{10,12}$ Neither of the above processes is accounted for in the hTST, since the latter applies to a given transition (and not to a distribution of transitions) and it is a harmonic theory, therefore neglecting multiphonon processes. ${ }^{10,13,14}$ From Eq. (2), the attempt frequency is rather interpreted as a topographic property of the potential energy surface related through the eigenfrequencies to the local curvature in the immediate vicinity of the initial and activated states. The Meyer-Neldel rule may therefore not apply within the hTST and, if it does, we may expect that it would appear as an additional process appearing in Eq. (3) as a dependence of $v_{\text {att }}^{0}$ on $E_{A}$. The presence or absence of the Meyer-Neldel rule within the hTST has been tested in crystals but the conclusions are not clear since it was found to apply in some cases ${ }^{15,16}$ and not in others. ${ }^{14}$

We investigate within the classical hTST the relation between the attempt frequency and the activation energy 

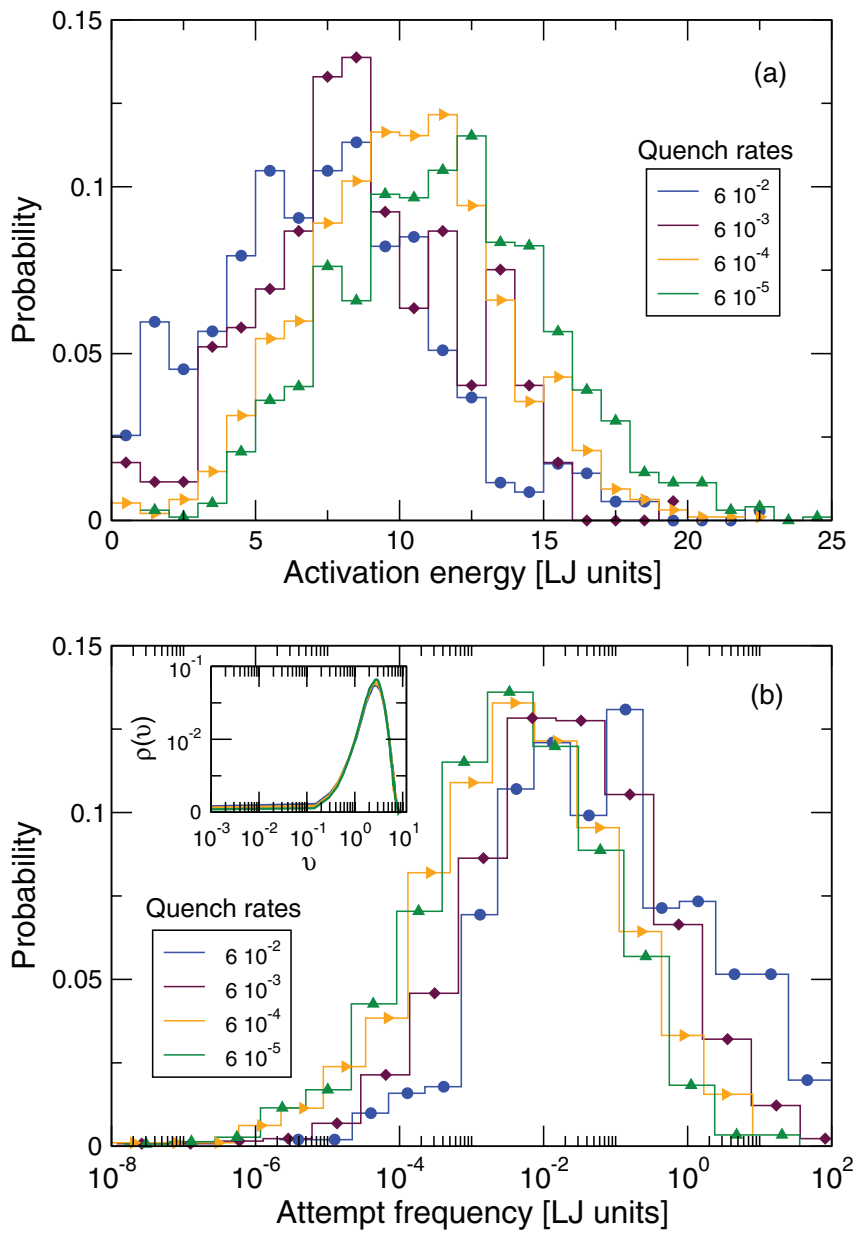

FIG. 1. (Color online) Distributions of (a) activation energies and (b) attempt frequencies in glasses quenched at different rates. The inset in (b) shows the distribution of eigenfrequencies (vibrational density of states) in the initial quenched glasses.

of thermally activated events in a Lennard-Jones glass. We considered an equimolar mixture of 4000 atoms interacting with the well-known Wahnström potential. ${ }^{17}$ Usual reduced units are used throughout the paper: $\epsilon_{A A}, \sigma_{A A}$, and $\tau_{A A}=$ $\sigma_{A A} \sqrt{m_{A} / \epsilon_{A A}}$. Glasses of density 1.2 were prepared by quenching a high-temperature liquid at different quench rates. Quenches were performed at constant volume with periodic boundary conditions (for details, see Ref. 18). The quench rate ranged from $6 \times 10^{-2}$ down to $6 \times 10^{-5} \tau_{A A}^{-1}$. The potential energy landscape of the quenched glasses was explored using ART, as implemented in Ref. 19. Random searches were iterated until at least 1000 distinct saddles were found for each configuration. Eigenfrequencies were then determined by diagonalizing the Hessian matrix of the four initial configurations and of each activated state. ${ }^{20}$

Figure 1 shows distributions of activation energies and attempt frequencies. Distributions of activation energies have the usual shape $e^{6,7,18,19}$ with a systematic trend: glasses quenched more slowly have a smaller density of low activation energies, or, in other words, distributions of activation energies shift towards higher energies in better-relaxed glasses. This effect is expected from a simple picture of the potential energy landscape where deeper energy minima are surrounded by higher activation energies.

Figure 1(b) shows the distributions of attempt frequencies. The range of attempt frequencies is very large, spanning ten orders of magnitude, in contrast with the usual assumption of a constant attempt frequency. By comparison with the distributions of eigenfrequencies (vibrational densities of states) in the initial quenched glasses shown in the inset of Fig. 1(b), attempt frequencies span a wider range than normal modes, in both the high- and low-frequency limits. Also, in contrast with the vibrational densities of states that are not strongly affected by the quench rate, ${ }^{21}$ the attempt frequencies show a clear trend: glasses quenched more slowly have lower attempt frequencies, or, in other words, distributions of attempt frequencies shift towards lower frequencies in better-relaxed glasses. For typical Lennard-Jones parameters, the time unit $\tau_{A A}$ is on the order of $1 \times 10^{-12}$ to $1 \times 10^{-13} \mathrm{~s}$. The higher frequencies in the distributions are therefore in the range of the usual estimate of attempt frequencies, but the rest of the distribution is much lower. In a related work, Kopsias and Theodorou ${ }^{22}$ found activation entropies spanning $10 k$, i.e., attempt frequencies spread over four orders of magnitude. The reason for this smaller range is presumably that the entropies were calculated on sequences of connected minima, rather than more exhaustive samplings from given configurations as done here.

We consider in Fig. 2 the relation between the activation energy of an event and its attempt frequency. This plot confirms the large energy and frequency ranges as well as the trends mentioned above; that is, activation energies shift towards higher energies and attempt frequencies towards lower frequencies in glasses quenched more slowly. Also, although scatters are large, we see that the logarithm of the attempt frequency tends to decrease linearly with increasing attempt frequency. We computed Pearson's product-moment correlation coefficient and found that it increases (in absolute value) with decreasing quench rates, indicating an increasingly clear linear relation in glasses quenched more slowly. A best fit of the data in Fig. 2 using an exponential function yields

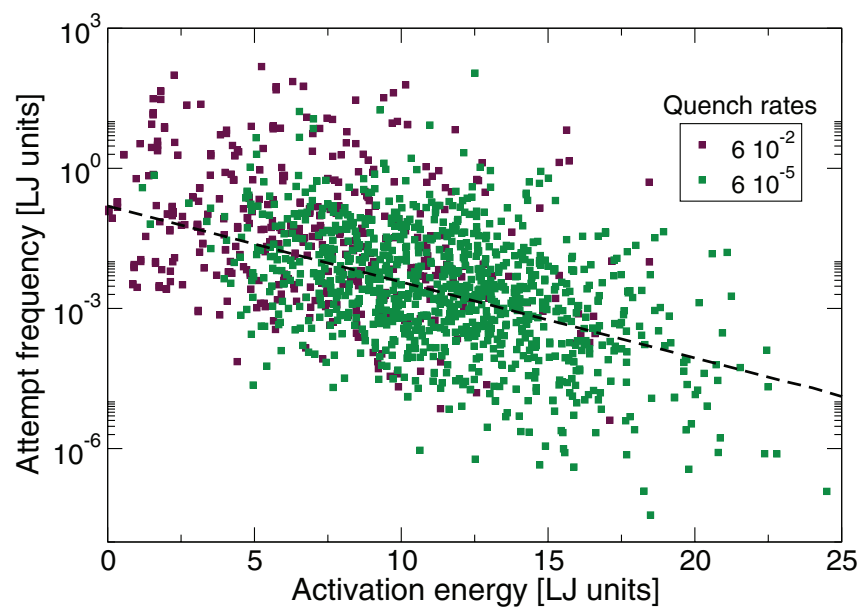

FIG. 2. (Color online) Relation between activation energy and attempt frequency within the hTST for thermally activated transitions in glasses quenched at different rates. For the sake of clarity, only two quench rates are shown. 
$v_{\text {att }}^{\mathrm{hTST}} \propto \exp \left(-E_{A} / k T_{1}\right)$ with $k T_{1} \sim 2.50$. Because of the large scatters, the uncertainty on $T_{1}$ is large, but, as an order of magnitude, $k T_{1}$ is systematically larger than 1 .

This dependence is opposed to the usual Meyer-Neldel rule, where the attempt frequency increases exponentially with the activation energy. However, as explained above, the present dependence is distinct from the effect of multiple particle hops and/or multiple thermal excitations, yielding an effective attempt frequency expected to be proportional to $\exp \left(E_{A}\left(1 / k T_{0}-1 / k T_{1}\right)\right)$. The characteristic temperature $T_{0}$ has been related to the glass transition temperature in the case of multiple hops ${ }^{11}$ (in the related "trap model" of glassy systems, ${ }^{23} T_{0}$ corresponds exactly to the glass transition temperature of the model), i.e., close to the mode-coupling temperature (here $T_{C}=0.59$ ), and to two to three times the Debye temperature in case of multiphonon excitations, ${ }^{10,12,15}$ which can be estimated from the maximum normal mode frequency, $T_{D} \sim 0.05$, with $v_{\max }=10$ and $\tau_{A A}=10^{-12} \mathrm{~s}$. In both cases, $T_{1}>T_{0}$, yielding a globally positive Meyer-Neldel rule, with a corrected prefactor.

We note that in Figs. 1 and 2, there is a single initial configuration for each quench rate. Therefore, the curvature term in Eq. (2) from the initial configuration (numerator) is constant for a given quench rate and the variations of the attempt frequencies reflect only variations of the curvature at the activated state. A decrease of the attempt frequency with increasing activation energy thus implies that saddles with a higher activation energy have a larger average curvature. We checked the generality of the very large scatters in attempt frequencies and of the inverse Meyer-Neldel rule by performing the same analysis for several glasses at each quench rate as well as for glasses modeled with the Kob-Andersen potential.

The dependence of the attempt frequency on the activation energy and the apparent opposition to the Meyer-Neldel rule reported above depend on the process studied. As an illustration, we consider in Fig. 3 a glass approaching a plastic instability, a classical situation encountered in athermal

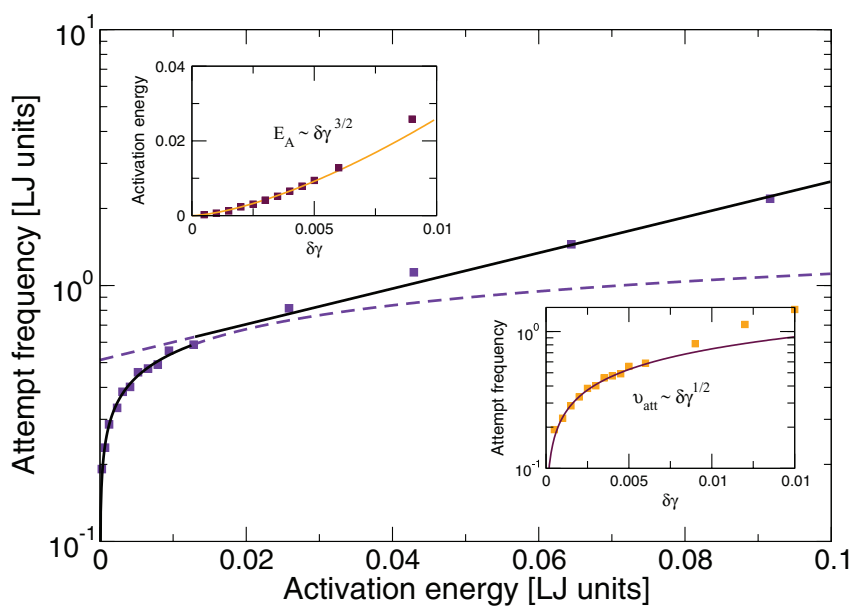

FIG. 3. (Color online) Relation between activation energy and attempt frequency near a plastic instability in a sheared glass (which corresponds to $E_{A}=0$ ). The insets show the dependence of the activation energy and attempt frequency on the distance to instability. quasistatic plasticity. ${ }^{24}$ We deformed a glass in an initial IS through increments of simple shear $\gamma$ separated by energy quenches until an instability to a new IS was reached. The glass was unloaded to generate a succession of matching configurations in the initial and final ISs at different applied strains. The nudged elastic band (NEB) method ${ }^{25}$ was then used to determine the activated states, from which activation energies and attempt frequencies were computed as a function of the distance to the critical strain, $\delta \gamma=\gamma_{C}-\gamma$. As seen in Fig. 3, the attempt frequency decreases with the activation energy near the instability with an exponential dependence for activation energies above about 0.02, in accordance with the usual Meyer-Neldel rule. The attempt frequency decreases partly because of a slow increase of the curvature at the saddle point (in contrast with the trend mentioned above) but mostly because, the instability being a fold bifurcation, an eigenvalue in the initial configuration goes to zero as $\delta \gamma^{1 / 2} \cdot{ }^{26}$ The insets in Fig. 3 show separately the dependence of the activation energy and attempt frequency on the distance to instability. The expected scaling law for the activation energy is recovered, $E_{A} \propto \delta \gamma^{3 / 2} \cdot{ }^{26}$ For the attempt frequency, if the decrease was solely dominated by the lowest eigenvalue in the initial configuration, an exponent of $1 / 4$ would be obtained since eigenfrequencies are square roots of eigenvalues. The effective exponent of $1 / 2$ found here (see the inset) is caused by several eigenfrequencies varying near the instability. Interestingly, we recover the same exponent, 1/2, predicted by Kramer's theory, ${ }^{26}$ although in this case, half of the exponent comes from the decay of the negative curvature at the saddle point, which is not accounted for in the hTST.

The large scatters reported above do not rule out the possibility to describe the kinetics of glasses with a single activation energy and attempt frequency, at least at low temperature. We show in Fig. 4 the hTST estimate of the total escape rate for the four configurations quenched at different rates:

$$
\Gamma_{\text {tot }}=\sum_{\text {sampling }} v_{\text {att }}^{\mathrm{hTST}} \exp \left(-E_{A} / k T\right) .
$$

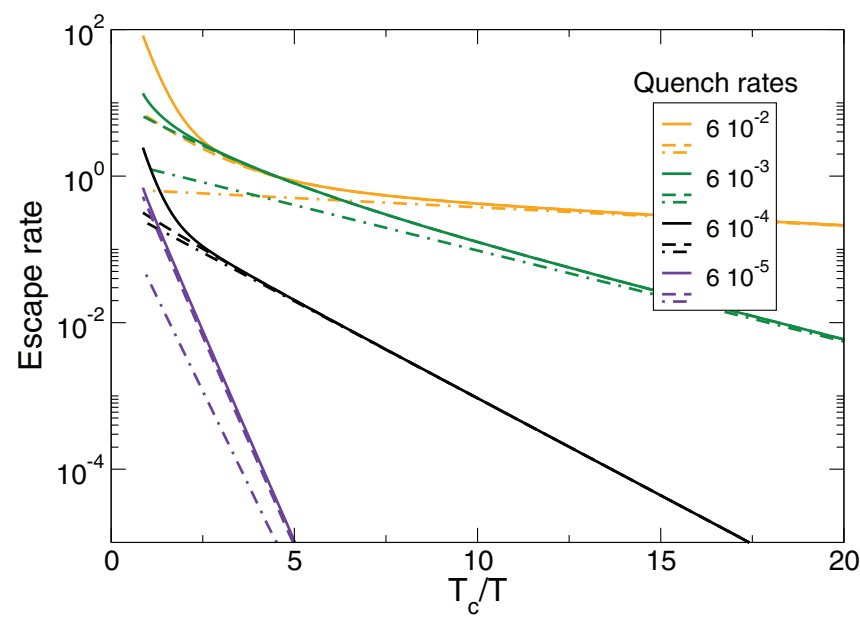

FIG. 4. (Color online) Prediction of the escape rate in glasses quenched at different rates. The fits were obtained using only the lowest energy transition (dash-dotted lines) or the transitions with $k T_{C}$ from the lowest energy transition (dashed lines). 
There is an Arrhenius regime at low temperature, starting at a temperature which increases in better-relaxed glasses. Two fits were added: the dash-dotted lines were obtained by limiting the calculation of the escape rate to the lowest energy transition, while the dashed lines include the transitions within $k T_{C}$ from the lowest energy transition. Just using the lowest energy transition already provides a rather satisfactory agreement with the full calculations, showing that at low temperatures only the very low energy transitions matter. The reason is that the distributions of activation energies in Fig. 1(a) do not increase fast enough at low energies, and are penalized by the corresponding decreasing attempt frequencies, to give a non-negligible weight to transitions with activation energies significantly larger than the minimum.

At low temperatures, the effective activation energy thus reduces to the lowest activation energy of the samples. Predicting an effective attempt frequency is more difficult because we have to account for a degeneracy factor, i.e., the number of transitions within $k T_{C}$. This effect is most visible for the best-relaxed glass where the prediction from a single transition is a factor between 5 and 10 less than the full calculation. We should also keep in mind that the present rates are only estimates since the samples are necessarily incomplete. Large variations in the low-temperature rates of Fig. 4 were observed depending on the number and properties of low-energy saddles contained in the samples. No quantitative trend can therefore be concluded from this figure. However, we mention that, for the specific system studied here, a direct MD trajectory was found to visit saddle points on the potential energy surface that all lie within the range sampled by the ART calculation. ${ }^{27}$

In conclusion, our work shows that a glass contains very wide ranges of activation energies and attempt frequencies that are probably characteristic of fragile glasses, which contain diverse atomic environments. By way of contrast, in strong glasses, where topological constraints are stronger and lead to fewer different atomic environments, distributions of activation energies and attempt frequencies are narrower, as reported, for instance, in silicon glasses. ${ }^{6}$ It was also found that the dynamics at low temperatures is controlled by the lowest energy transitions, which have high frequencies close to the Debye frequency. This justifies the usual assumption of a single activation energy but also shows that predicting the effective attempt frequency requires that we account for a degeneracy factor, which is difficult to evaluate without an exhaustive sampling of the potential energy landscape near the minimum energy transition.
${ }^{1}$ D. Rodney, A. Tanguy, and D. Vandembroucq, Modell. Simul. Mater. Sci. Eng. 19, 083001 (2011).

${ }^{2}$ T. B. Schrøder, S. Sastry, J. C. Dyre, and S. C. Glotzer, J. Chem. Phys. 112, 9834 (2000).

${ }^{3}$ H. Eyring, J. Chem. Phys. 3, 107 (1935).

${ }^{4}$ G. H. Vineyard, J. Phys. Chem. Solids 3, 121 (1957).

${ }^{5}$ G. T. Barkema and N. Mousseau, Phys. Rev. Lett. 77, 4358 (1996).

${ }^{6}$ F. Valiquette and N. Mousseau, Phys. Rev. B 68, 125209 (2003).

${ }^{7}$ H. Kallel, N. Mousseau, and F. Schiettekatte, Phys. Rev. Lett. 105, 045503 (2010).

${ }^{8}$ D. Rodney and C. Schuh, Phys. Rev. Lett. 102, 235503 (2009).

${ }^{9}$ W. Meyer and H. Neldel, Z. Tech. Phys. (Leningrad) 12, 588 (1937).

${ }^{10}$ A. Yelon, B. Movaghar, and R. S. Crandall, Rep. Prog. Phys. 69, 1145 (2006).

${ }^{11}$ J. C. Dyre, J. Phys. C 19, 5655 (1986).

${ }^{12}$ G. Boisvert, L. J. Lewis, and A. Yelon, Phys. Rev. Lett. 75, 469 (1995).

${ }^{13}$ G. Boisvert, N. Mousseau, and L. J. Lewis, Phys. Rev. B 58, 12667 (1998).

${ }^{14}$ M. C. Marinica, C. Barreteau, D. Spanjaard, and M. C. Desjonquères, Phys. Rev. B 72, 115402 (2005).
${ }^{15}$ G. Boisvert and L. J. Lewis, Phys. Rev. B 54, 2880 (1996).

${ }^{16}$ O. N. Bedoya-Martinez and G. Roma, Phys. Rev. B 82, 134115 (2010).

${ }^{17}$ G. Wahnström, Phys. Rev. A 44, 3752 (1991).

${ }^{18}$ D. Rodney and T. Schrøder, Eur. Phys. J. E 34, 100 (2011).

${ }^{19}$ D. Rodney and C. A. Schuh, Phys. Rev. B 80, 184203 (2009).

${ }^{20}$ Intel Math Kernel Library (MKL), http://software.intel.com/ en-us/intel-mkl.

${ }^{21}$ P. M. Derlet, R. Maaß, and J. F. Löffler, Eur. Phys. J. B 85, 148 (2012).

${ }^{22}$ N. Kopsias and D. Theodorou, J. Chem. Phys. 109, 8573 (1998).

${ }^{23}$ J. P. Bouchaud, J. Phys. France 2, 1705 (1992).

${ }^{24}$ C. Maloney and A. Lemaître, Phys. Rev. Lett. 93, 195501 (2004).

${ }^{25}$ G. Henkelman, B. P. Uberuaga, and H. Jónsson, J. Chem. Phys. 113, 9901 (2000).

${ }^{26}$ C. E. Maloney and D. J. Lacks, Phys. Rev. E 73, 061106 (2006).

${ }^{27}$ The MD simulation included 300 trajectories starting from the same minimum, and the saddle point analysis was carried out by quenching regularly and analyzing the saddle point structure with the NEB method when a change of minimum was identified. 